UOSTP-03-104

hep-th/0308027

\title{
Holography with Timelike Bulk Hypersurfaces
}

\author{
Dongsu Bak* \\ Physics Department, University of Seoul, Seoul 130-743, Korea
}

\begin{abstract}
We propose a new version of holographic principle. This proposal extends the holographic principle based on the lightsheet to the one constraining the entropy passing through bulk hypersurface of timelike geodesics by the boundary area divided by $4 G$. We give a proof of the proposal in the classical regime based on a simple local entropy condition.
\end{abstract}

November 9, 2018

*email: dsbak@mach.uos.ac.kr 


\section{Introduction}

The holographic principle may be a key paradigm for the understanding of the true nature of gravity and string theories. As suggested first by 't Hooft and Susskind, it states that the degrees of freedom for the gravitational system reside not in the bulk but in its boundaries [1, 2]. Furthermore the number of boundary degrees of freedom per Planck area is constrained not to exceed unity. This principle clearly contradicts with the current framework of quantum field theories because their numbers of degrees scale like the bulk volume not by the boundary area. These ideas are precisely exemplified in the formulation of AdS/CFT correspondence 3, 4. When the gravity and field theories coexist like our Universe, the UV structure of quantum field theories must be modified drastically in order to have fundamental degrees limited by their boundary areas.

Application of the above holographic principle to more general spacetime including cosmology is problematic [5]. For the flat FRW universe as an example, the matter entropy scales like the coordinate volume and the bulk entropy eventually wins over the area bound for large enough region. The authors of [5] suggested that the entropy crossing past lightlike region generated from the particle horizon should be bounded by the area of the particle horizon. Based on the subsequent works [6], Bousso has given a covariant version of the holographic principle that may be applicable for general backgrounds [7]. It states that the entropy on the lightsheet $L$ is bounded by its boundary area divided by $4 G$. The lightsheet is defined by the bundle of lightlike geodesics that are orthogonally generated from the boundary surface with nonpositive expansion away from the boundary surface. The shape of the boundary surface may be arbitrary once it is consistent with the nonpositive expansion condition.

More stronger bound is proposed by Flanagan, Marolf and Wald (FMW) [8, asserting that the entropy flux passing through the lightsheet orthogonally generated from one boundary and ending on another boundary is bounded by the difference of the boundary areas divided by $4 G$. Interestingly this version can be proven in the semiclassical limit provided certain local conditions on the entropy are assumed [9, 10. The proof is utilizing focusing nature of the lightlike geodesic based on the Raychaudhuri equation.

In this note, we observe that the Raychaudhuri equation is also for the timelike geodesics, which plays an important role in the investigation of the global structures of spacetime including horizons

and singularities. We extend the above holography to the case where the bulk hypersurface consists of timelike geodesics. The proposal dictates that the entropy passing through the bulk hypersurface consisting of timelike geodesics orthogonally generated from a codimension two spacelike hypersur- 
face $B$ and terminating on $B^{\prime}$, should be bounded by the difference of the boundary area divided by $4 G$. The proof shall be given in the classical regime with simple well motivated local entropy conditions.

There is a strong supportive argument for the above timelike holography. Consider a $d+1$ dimensional geometry of the form, $M \times S^{1}$, where $M$ is a $d$ dimensional spacetime and $S^{1}$ denotes a circle. Lightlike geodesics in the $d+1$ dimensions correspond to timelike geodesics in the spacetime $M$ if the momentum along the circle is nonvanishing. This way the lightlike holography in $M \times S^{1}$ may lead to the timelike holography in the lower dimensional spacetime ${ }^{1}$.

This paper is organized as follows. In Section 2, we review the covariant holography proposed by Bousso and extended by FMW. In Section 3, we give the proof (in the classical regime) based on the simple local conditions on the entropy density. Section 4 deals with our proposal on the holography with timelike bulk hypersurface. We discuss a proof of the statement starting from appropriate local conditions on the entropy flux. Last section is devoted for the discussions.

\section{Review of the lightlike holographic principle}

For later use, we will review the basic construct of the holographic principle introduced by Bousso and later extended by FMW.

The Bousso's holographic principle compares the entropy on the lightsheet $L$ with its boundary area divided by $4 G$. The lightsheet is defined by the bundle of lightlike geodesics that are orthogonally generated from the boundary surface with nonpositive expansion away from a spacelikesurface. The principle then dictates that

$$
S_{L} \leq \frac{A}{4 G}
$$

where $S_{L}$ is the entropy flux passing through the lightsheet and $A$ the boundary area.

The lightsheet consists of collections of lightlike geodesics $k^{a}=\left(\frac{\partial}{\partial \lambda}\right)^{a}$ emanated orthogonally from a given codimension-two spacelike surface $B$ where $\lambda$ is the affine parameter. The expansion,

$$
\theta=\nabla_{a} k^{a}
$$

describes the changing rate of the logarithm of the cross sectional area of the light rays with respect to $\lambda$. The definition of lightsheet requires the expansion away from the boundary surface to be

\footnotetext{
${ }^{1}$ We like to thank Dominic Brecher for pointing out this argument. There are also discussions about the dimensional reduction and lightlike holography in Refs. [11, 12.
} 
nonpositive. The lightsheet should be terminated if the expansion turns into a positive value or the geodesics hit singularities.

Though the local concept of entropy is not well defined, we introduce the entropy current density $s^{a}$, which may have a well defined physical meaning in the thermodynamic limit. The entropy flux density $s$ passing through the lightsheet is given by

$$
s=n_{a} s^{a},
$$

with the vector $n_{a}=-k_{a}\left(+k_{a}\right)$ normal to the future (past) directed lightsheet. The total entropy passing through the lightsheet is the volume integral,

$$
S_{L}(B)=\int d y^{D-2} \sqrt{h(y)} \int d \lambda \mathcal{A} s,
$$

where $\vec{y}$ is the coordinate for the boundary and $h(y)$ is the determinant of the induced metric on the boundary. The area factor $\mathcal{A}$ represents the growth of the unit area along the affine parameter

$$
\mathcal{A}=\exp \left[\int_{0}^{\lambda} d \tilde{\lambda} \theta(\tilde{\lambda})\right],
$$

and the cross sectional area at $\lambda$ is then given by

$$
A(\lambda)=\int d y^{D-2} \sqrt{h(y)} \mathcal{A}(\lambda)
$$

More stronger bound,

$$
S_{L\left(B-B^{\prime}\right)} \leq \frac{1}{4 G}\left(A-A^{\prime}\right)
$$

has been proposed by FMW, where $S_{L\left(B-B^{\prime}\right)}$ corresponds to the entropy flux passing through the lightlike surface orthogonally generated from $B$ and ending on $B^{\prime}$ before reaching caustic or singularities. One compares this entropy to the difference of the area of $B$ and $B^{\prime}$. This version of the entropy bound is the one we are interested in this note.

\section{Conditions for the holography}

The latter version of the holography can be shown to hold in the classical regime with certain conditions on the entropy flux density are satisfied.

Here we review the conditions and the proof given recently in Refs. [9, 10. The entropy conditions used are:

$$
\text { i. } \quad s^{\prime} \leq 2 \pi T_{a b} k^{a} k^{b}
$$




$$
\text { ii. } s(0) \leq-\frac{1}{4 G} \theta(0)
$$

The second condition is on the initial condition for the entropy and the first corresponds to the local version of the Bekenstein bound as discussed in Ref. [9, 13, 14].

Based on these two conditions, the proof of the entropy bound is very simple in the semiclassical limit where one may use the Einstein equation,

$$
R_{a b}-\frac{1}{2} g_{a b} R=8 \pi G T_{a b}
$$

The expansion of the bundle of the lightlike geodesics obeys the Raychaudhuri equation

$$
\theta^{\prime}=-\frac{1}{D-2} \theta^{2}-\sigma_{a b} \sigma^{a b}+\omega_{a b} \omega^{a b}-R_{a b} k^{a} k^{b},
$$

where $\sigma_{a b}$ and $\omega_{a b}$ are the shear and the twist. Since the lightsheets are hypersurface orthogonal, the twist $\omega_{a b}$ vanishes.

The FMW bound has a differential form. We note that the local inequality,

$$
s \leq-\frac{1}{4 G} \theta
$$

implies the integral version (7). In particular considering $B^{\prime}$ infinitesimally away from $B$ in terms of the affine parameter, one gets the condition (ii) from the integral version. It is clear that the initial condition on the entropy should hold without any disposal because the part of statement of the holography is the initial condition itself. Since $s(0)$ is not to do with how the boundary surface is curved, the initial condition is not satisfied for an arbitrary boundary surface. Rather at a given initial spacetime point, one needs more curved boundary in order to have the negative of expansion larger than the initial entropy flux density. Therefore the initial condition puts restriction not on the initial entropy density but on the boundary surface. In Ref. [9], the authors insisted $\theta(0) \leq 0$, which is clearly different from ours.

From the two conditions, the proof of the inequality (10) is quite simple. Using (9), the first condition and the Einstein Equation, we have

$$
-\frac{1}{4 G} \theta^{\prime}=\frac{1}{4 G(D-2)} \theta^{2}+\frac{1}{4 G} \sigma_{a b} \sigma^{a b}+2 \pi T_{a b} k^{a} k^{b} \geq s^{\prime} .
$$

Hence together with the initial condition, one has

$$
s=s_{0}+\int_{0}^{\lambda} d \tilde{\lambda} s^{\prime}(\tilde{\lambda}) \leq-\frac{1}{4 G}\left(\theta_{0}+\int_{0}^{\lambda} d \tilde{\lambda} \theta^{\prime}(\tilde{\lambda})\right)=-\frac{1}{4 G} \theta .
$$


A few comments are in order. The first condition on the local change of the entropy is closely related to the Bekenstein bound as discussed recently in Ref. [9, 13, 14. We shall not repeat the arguments here. One might try using the following weaker condition;

$$
s^{\prime} \leq 2 \pi T_{a b} k^{a} k^{b}+\frac{1}{4 G(D-2)} \theta^{2} ?
$$

But the expansion is not such a physical quantity that is fixed solely by the spacetime point. Rather it is determined by the point as well as by the shape of the initial surface. On the other hand, $s^{\prime}$ and $T_{a b} k^{a} k^{b}$ are the physical quantities (of course depending on the geodesic $k^{a}$ ) that are nothing to do with the shape of the initial surface. In this respect we view that the relation (13) is not desirable.

The saturation of the above holographic principle requires $\theta=0$ as well as $s^{\prime}=2 \pi T_{a b} k^{a} k^{b}$. The $\theta=0$ condition is particularly strict. For example in the case of AdS geometries, we do not have holographic screen that saturates the holographic bound. The reason is because the $\theta=0$ condition cannot hold along the lightsheet. Let us take a lightsheet in the IR region of AdS space, for which the spacelike projection theorem is applicable. Then the lightsheet records all the entropy of the bulk inside. As discussed in detail in Ref. [4, the boundary area of $\operatorname{AdS}_{5} \times S^{5}$ divided by $4 G$ located at the IR region of AdS space agrees with the degrees of freedom count of $N=4$ SYM theory up to order one numerical constant. However, if the current formulation of the holographic principle is correct, the numerical coefficient cannot be unity because the bound cannot be saturated. Namely $S_{B u l k}=S_{C F T} \leq S_{L}<\frac{1}{4 G} A$ with $S_{B u l k}$ and $S_{C F T}$ being respectively the entropies of the bulk and the boundary $N=4 \mathrm{SYM}$ theory. Since the original conjecture is the equivalence between gravity with $N=4 \mathrm{SYM}$ theory in the large $\mathrm{N}$ limit, the non saturation is not a problem.

Let us give here examples where the bound is saturated. A rather trivial example is the case of flat boundary in the flat spacetime. The expansion and the entropy flux density along the lightsheet remain zero. The bound is saturated because the both sides are zero. Another is the spacetime of a stationary black hole, where we take the event horizon as lightsheet. There the expansion stays zero. Hence the difference of the boundary areas is again zero. Also $s=0$ because nothing crosses the horizon due to the stationary nature of the black hole. Of course, we ignore the possible quantum corrections including the effect of Hawking radiation.

Finally we illustrate the use of holography for the spacetime representing the formation of black hole by the gravitational collapse of star. The relevant Penrose diagram is depicted in Fig. 1. Ignoring any quantum effect, the outside of the surface of the star is same as the vacuum Schwarzschild black hole solution with the horizon radius, say, $R_{S}$. The event horizon (the line with arrow) begins with $r=0$, its radius grows toward the future infinity $i^{+}$and reaches the 


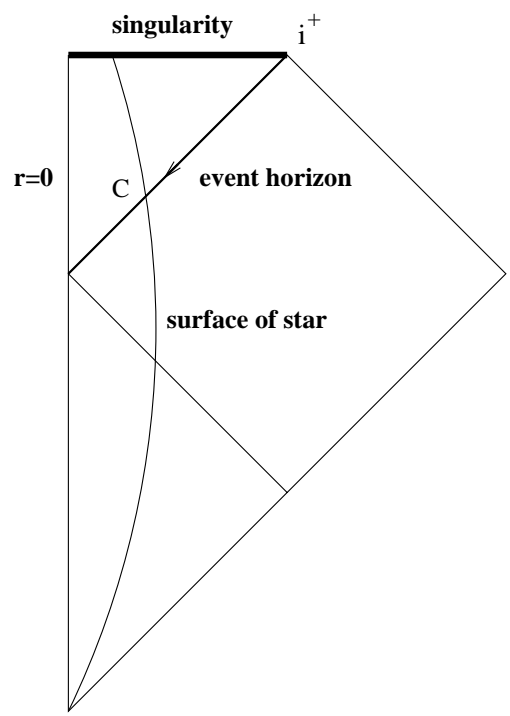

Figure 1: We depict the Penrose diagram of the spacetime representing the formation of the black hole by the gravitational collapse of a star.

stationary value $R_{S}$ once the surface of star is crossed. The increase is monotonic in accordance with the theorem of horizon area for the matter content of the star satisfying the weak energy condition. Let us consider the lightsheet starting from somewhere between $i^{+}$and $C$ and ending up at $r=0$. (Thus $\lambda=0$ at the starting point.) Expansion is nonpositive everywhere. In fact $\theta=0$ at $\lambda=0$ since the radius (or area) is not changing there. At the world point $C$, it becomes negative and keeps decreasing until reaching negative infinity at the caustic of $r=0 .\left(\theta^{\prime} \leq 0\right.$ all the way once the weak energy condition is satisfied.) The initial entropy condition is satisfied because $\theta(0)=s(0)=0$. Using the holography bound, we conclude that

$$
S_{S}<S_{B}=\frac{1}{4 G} A_{B}
$$

where $S_{S}$ is the entropy flux of the star through the horizon and $S_{B}\left(A_{B}\right)$ is the entropy (the horizon area) of the final Schwarzschild black hole. The equality is not possible because $\theta$ is nonzero somewhere. The interpretation of the result here is that the entropy of star used for the black hole formation is less than the final (gravitational) entropy of the black hole. In other words there is a positive amount of entropy production along the gravitational collapse.

\section{Holography with timelike sheets}

In the discussion of the holography with lightsheets, the Raychaudhuri equation plays an important role. The Raychaudhuri equation is, however, not just for the bundle of lightlike geodesics but also 
for timelike geodesics. In this respect here we discuss whether one may extend the formulation of holography to the case of timelike bulk hypersurface consisting of timelike geodesics.

Let us consider bundle of timelike geodesics orthogonally generated from a codimension two spacelike hypersurface $B$ and terminating on $B^{\prime}$ before reaching caustics or singularities. We shall denote the timelike geodesic by $\xi^{a}=(\partial / \partial \tau)^{a}$ (with $\xi_{a} \xi^{a}=-1$ ) where $\tau$ is the proper time along the geodesic. We introduce the entropy flux density $s$ by

$$
s=n_{a} s^{a}
$$

where $n_{a}$ is the normal vector to the timelike bulk hypersurface. The entropy on the timelike bulk hypersurface is then

$$
S_{T\left(B-B^{\prime}\right)}=\int d y^{D-2} \sqrt{h(y)} \int_{0}^{\tau^{\prime}} d \tau \mathcal{B} s
$$

where $\vec{y}$ is the coordinate for the boundary and $\mathcal{B}$ represents the growth of the unit area along $\tau$ given by

$$
\mathcal{B}=\exp \left[\int_{0}^{\tau} d \tilde{\tau} \theta_{A}(\tilde{\tau})\right]
$$

with the area expansion $\theta_{A}$. Note that there are two possible choices for the normal vector field $n_{a}$. We shall always take the one that makes $S_{T\left(B-B^{\prime}\right)}$ nonnegative. Infinitesimally away from the timelike hypersurface to the normal direction, we extend the geodesics such that geodesics are still hypersurface orthogonal. The hypersurface orthogonality condition is rather strict and fix the extension in the infinitesimal region. Note that, in the timelike case, $\theta=\nabla_{a} k^{a}$ describes not the area expansion but the volume expansion rate. We introduce the coordinate vector $m^{a}=(\partial / \partial s)^{a}$ whose direction agrees with $n^{a}$ at the point on the timelike surface where we evaluate the expansion rate. By straightforward computation[15], one may show that

$$
\theta=\theta_{A}+\theta_{m}
$$

where $\theta_{m}$ denotes the expansion of the normal vector, $\frac{d}{d \tau} \ln |m|$. The timelike bulk hypersurface is further restricted by the initial condition

$$
s(0) \leq-\frac{1}{4 G} \theta(0),
$$

and the condition on the expansion of the vector $m^{a}$

$$
\theta_{m}(\tau) \geq 0
$$

We shall call the resulting hypersurface as "timelike sheet". At first sight, the latter condition appears too restrictive. However, we note that the effect of tidal force in general makes size of body grow when it is attracted toward a distribution of mass. This implies that $\theta_{m}$ is nonnegative 
rather generically for the geodesics of massive objects attracted toward the mass distribution. We shall give detailed examples of this elsewhere 15.

Now the proposal is simple. The entropy passing through the timelike sheet should be constrained by the difference of the boundary area divided by $4 G$;

$$
S_{T\left(B-B^{\prime}\right)} \leq \frac{1}{4 G}\left(A-A^{\prime}\right)
$$

where $T\left(B-B^{\prime}\right)$ denotes the timelike sheet.

For the proof (valid in the classical regime), we shall use the local entropy condition

$$
\text { iii. } \dot{s} \leq 2 \pi\left(T_{a b} \xi^{a} \xi^{b}+\frac{1}{D-2} T\right)
$$

where dots represent derivatives with respect to $\tau$ and $T=T_{a}^{a}$. As in the lightlike case, the initial condition (18) is not for the initial entropy flux but for the choice of the initial boundary surface.

The holography here is much stronger version than the previous ones, and implies the lightlike holography we discussed before. Namely if the holography is used for the timelike sheet that consists of almost lightlike geodesics, the lightlike holography follows as the limiting case. Therefore the timelike holography needs a stronger requirement on the local entropy flux. Considering the almost lightlike geodesics, the expansion of the normal vector, $\theta_{m}$, approaches zero and the condition (iii) implies the condition (i).

The proof of the above in the classical regime is straightforward. The Raychaudhuri equation reads

$$
\dot{\theta}=-\frac{1}{D-1} \theta^{2}-\sigma_{a b} \sigma^{a b}+\omega_{a b} \omega^{a b}-8 \pi G\left(T_{a b} \xi^{a} \xi^{b}+\frac{1}{D-2} T\right),
$$

where we used the Einstein equation. Since geodesics are hypersurface orthogonal, the twist $\omega_{a b}$ is zero automatically.

Again we note that the differential form of the holography,

$$
s \leq-\frac{1}{4 G} \theta \leq-\frac{1}{4 G} \theta_{A},
$$

implies the integral version, where the second inequality follows from the condition (19).

Using (21) and the condition (iii), we have

$$
-\frac{1}{4 G} \dot{\theta}=\frac{1}{4 G(D-1)} \theta^{2}+\frac{1}{4 G} \sigma_{a b} \sigma^{a b}+2 \pi\left(T_{a b} k^{a} k^{b}+\frac{1}{D-2} T\right) \geq \dot{s} .
$$

Together with the initial condition, one has

$$
s=s_{0}+\int_{0}^{\tau} d \tilde{\tau} \dot{s}(\tilde{\tau}) \leq-\frac{1}{4 G}\left(\theta_{0}+\int_{0}^{\tau} d \tilde{\tau} \dot{\theta}(\tilde{\tau})\right)=-\frac{1}{4 G} \theta .
$$


Like the case of the lightlike holography, so called spacelike projection theorem holds if certain conditions are met[7]. If the future directed timelike bulk hypersurface is complete i.e. $B$ is the only boundary, the entropy on the spacelike bulk enclosed by the boundary would be limited by the boundary area divided by $4 G$. This is a consequence of the generalized second law. Therefore if any future directed timelike sheet is complete, the original 't Hooft version of the holographic principle follows.

Main difference between the lightlike and the timelike holography lies in the form of the relevant energy conditions. For the lightlike case, the null energy condition,

$$
T_{a b} k^{a} k^{b} \geq 0 \quad \text { for all null } k^{a}
$$

is relevant. Let us consider a diagonalizable energy-momentum tensor that takes the form

$$
T_{a b}=\rho t_{a} t_{b}+\sum_{i=1}^{D-1} p_{i} x_{a}^{(i)} x_{b}^{(i)},
$$

where $\left(t_{a}, x_{a}^{(i)}\right)$ is an orthonormal basis with $t_{a}$ timelike and $x_{a}^{(i)}$ spacelike. Then the null energy condition implies

$$
\rho+p_{i} \geq 0 \text { for } i=1, \cdots, D-1
$$

On the other hand, the energy condition related to the the timelike holography is the strong energy condition

$$
T_{a b} \xi^{a} \xi^{b}+\frac{1}{D-2} T \geq 0 \quad \text { for all timelike } \xi^{a} .
$$

For the above form of the energy momentum tensor, the strong energy condition implies

$$
(D-3) \rho+\sum_{i} p_{i} \geq 0 \text { and } \rho+p_{i} \geq 0 \text { for } i=1, \cdots, D-1 .
$$

It is generically believed that, for any classically reasonable matter, the energy density $T_{a b} \xi^{a} \xi^{b} \geq 0$ for any timelike vector $\xi^{a}$. This energy condition is referred as weak energy condition. In term of the energy density and momentum, the weak energy condition implies

$$
\rho \geq 0 \text { and } \rho+p_{i} \geq 0 \text { for } i=1, \cdots, D-1 .
$$

The strong energy condition is violated for the energy momentum tensor of positive cosmological constant. Due to the relation $\rho=-p_{i}>0$, one of the strong energy condition is violated by $(D-3) \rho+\sum_{i} p_{i}=-2 \rho<0$.

The null energy or the strong energy conditions are not necessary conditions for the lightlike or the timelike holography because the derivative of entropy flux density may be positive or negative. 


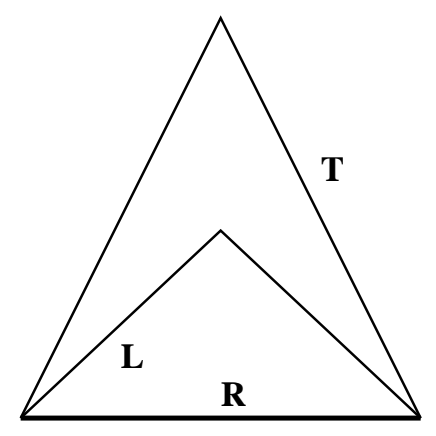

Figure 2: Here we consider the case where the timelike-sheet is complete such that the spacelike projection theorem is applicable. The solid line at the bottom represents the spacelike region enclosed by some closed boundary. $L$ and $T$ are respectively for the lightsheet and the timelike-sheet.

Finally let us illustrate one specific example in which the timelike holography leads to a stronger statement than the lightlike holography. We take the case where the future-directed timelike sheet is complete before reaching singularity with the initial conditions on the expansion respected. (See Fig. 2.) Using the lightlike and the timelike holography, one has $S_{L} \leq \frac{1}{4 G} A$ and $S_{T} \leq \frac{1}{4 G} A$ with $A$ being the boundary area. In addition, by the generalized second law, $S_{L} \leq S_{T}$. Thus, one gets

$$
S_{L} \leq S_{T} \leq \frac{1}{4 G} A
$$

This is clearly a stronger statement than the one from the lightlike holography i.e. $S_{L} \leq \frac{1}{4 G} A$.

\section{Discussions}

In this note, we propose a new version of holographic principle which compares the entropy passing through a bulk hypersurface consisting of timelike geodesics generated from a boundary surface to the boundary area divided by $4 G$. We discuss the corresponding entropy condition closely related to the Bekenstein bound as a generalization of the lightlike case. Based on the condition, we prove our proposal in the classical regime. It may be quite interesting to derive our timelike holography from the lightlike holography of one higher dimensions via the dimensional reduction with a nonvanishing Kaluza-Klein momentum.

Our proposal is stronger than the lightlike case because the lightlike case can be obtained by a limiting procedure in which the velocity field along the timelike geodesics approaches the light velocity arbitrarily closely.

One may wonder whether further extension of the holography to the one based on spacelike geodesics is possible or not. However the spacelike version, if possible, will lead to the 't Hooft 
version of holography for general backgrounds, for which we already gave the flat FRW universe as a counterexample.

There are number of points to be studied further. Here we do not discuss about the possible quantum modification of the timelike holographic principle. In the lightlike case, an attempt is made in Ref. [10. The Raychaudhuri equation also plays an essential role for the proof of the singularity theorem. As we discussed, the presence of singularity limits lightsheets and timelike sheets. Singularities seem to have some connections with the entropy of the system. In presence of the cosmological singularities like the big bang, the meaning of the generalized second law is not quite clear. Namely the entropy of certain region may not have any operational meaning because the part of the region may lie outside of the observer's particle horizon. Moreover, the notion of time loses its meaning at the big bang or other singularities. Can we talk about then the fate of degrees of freedom or entropy there? The holographic principle may shed some light on how to tackle the singularities.

\section{Acknowledgments}

We are grateful to Raphael Bousso and Naoki Sasakura for useful discussions and conversations. This work is supported in part by KOSEF 1998 Interdisciplinary Research Grant 98-07-02-07-01-5.

\section{References}

[1] G. 't Hooft, "Dimensional Reduction In Quantum Gravity," arXiv:gr-qc/9310026.

[2] L. Susskind, "The World as a hologram," J. Math. Phys. 36, 6377 (1995) arXiv:hep-th/9409089.

[3] J. M. Maldacena, "The large N limit of superconformal field theories and supergravity," Adv. Theor. Math. Phys. 2, 231 (1998) [Int. J. Theor. Phys. 38, 1113 (1999)] arXiv:hep-th/9711200.

[4] L. Susskind and E. Witten, "The holographic bound in anti-de Sitter space," arXiv:hep-th/9805114

[5] W. Fischler and L. Susskind, "Holography and cosmology," arXiv:hep-th/9806039.

[6] D. Bak and S. J. Rey, "Cosmic holography," Class. Quant. Grav. 17, L83 (2000) arXiv:hep-th/9902173; R. Easther and D. A. Lowe, "Holography, cosmology and the second law of thermodynamics," Phys. Rev. Lett. 82, 4967 (1999) arXiv:hep-th/9902088; 
G. Veneziano, "Pre-bangian origin of our entropy and time arrow," Phys. Lett. B 454, 22 (1999) arXiv:hep-th/9902126; N. Kaloper and A. D. Linde, "Cosmology vs. holography," Phys. Rev. D 60, 103509 (1999) arXiv:hep-th/9904120.

[7] R. Bousso, "A Covariant Entropy Conjecture," JHEP 9907, $004 \quad$ (1999) arXiv:hep-th/9905177.

[8] E. E. Flanagan, D. Marolf and R. M. Wald, "Proof of Classical Versions of the Bousso Entropy Bound and of the Generalized Second Law," Phys. Rev. D 62, 084035 (2000) arXiv:hep-th/9908070.

[9] R. Bousso, E. E. Flanagan and D. Marolf, "Simple sufficient conditions for the generalized covariant entropy bound," arXiv:hep-th/0305149.

[10] A. Strominger and D. M. Thompson, "A quantum Bousso bound," arXiv:hep-th/0303067.

[11] E. K. Boyda, S. Ganguli, P. Horava and U. Varadarajan, "Holographic protection of chronology in universes of the Goedel type," Phys. Rev. D 67, 106003 (2003) arXiv:hep-th/0212087.

[12] D. Brecher, P. A. DeBoer, D. C. Page and M. Rozali, "Closed timelike curves and holography in compact plane waves," arXiv:hep-th/0306190

[13] J. D. Bekenstein, "Generalized Second Law Of Thermodynamics In Black Hole Physics," Phys. Rev. D 9, 3292 (1974).

[14] R. Bousso, "Light-sheets and Bekenstein's bound," Phys. Rev. Lett. 90, 121302 (2003) arXiv:hep-th/0210295.

[15] Dongsu Bak, Seok Kim and Ho-Ung Yee, in preparation. 